\title{
SITUATION ASSESSMENT IN DISASTER MANAGEMENT
}

\author{
JUAN CARLOS AUGUSTO, HUI WANG, JUN LIU \\ School of Computing and Mathematics and Computer Science Research Institute, \\ University of Ulster, Jordanstown, Co. Antrim, BT370QB, U.K. \\ E-mail: [jc.augusto, h.wang, j.liu]@ulster.ac.uk
}

Received: 11-01-2008

Revised: $\quad 30-06-2008$

\begin{abstract}
We present a framework for decision-making in relation to disaster management with a focus on situation assessment during disaster management monitoring. The use of causality reasoning based on the temporal evolution of a scenario provides a natural way to chain meaningful events and possible states of the system. There are usually different ways to analyse a problem and different strategies to follow as a solution and it is also often the case that information originating in different sources can be inconsistent or unreliable. Therefore we allow the specification of possibly conflicting situations as they are typical elements in disaster management. A decision procedure to decide on those conflicting situations is presented which not only provides a framework for the assistance of one decision-maker but also how to handle opinions from a hierarchy of decision-makers.
\end{abstract}

\section{Introduction}

Disaster management, which encompasses monitoring, predicting, preventing, preparing for, responding to, mitigating and recovering from disasters, aims to deal with any potential and actual disaster by effective and efficient organization, communication, interaction and utilization of counter-disaster resources.

Decisions taken in relation to a disaster can alter its development and its consequences dramatically. Usually decisions are made by humans but more and more computer-based decision-making support-systems have been developed and deployed.

Although human decision-makers (DMs) are usually better than machines to judge complex situations and make thoughtful decisions, computers can provide a stress-free view of the situation, make efficient use of a broad range of important knowledge, calculated decisions.
Our intelligent mixed-initiative system has the overall architecture portrayed in Figure 1.

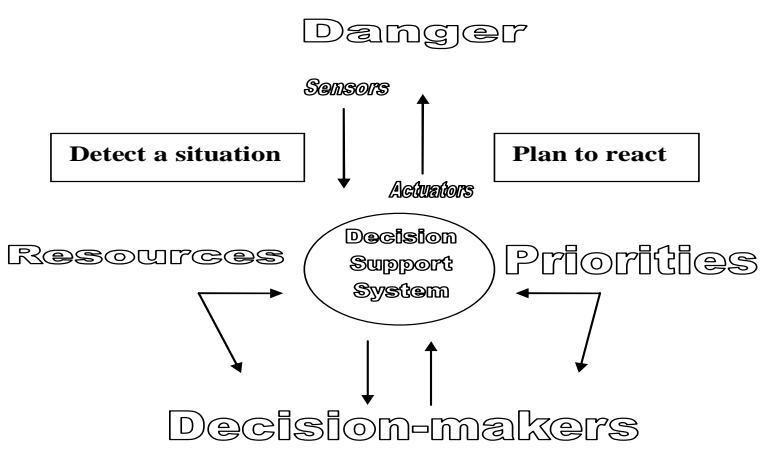

Fig. 1. General mixed-initiative architecture.

The architecture is general enough to be applicable to different types of environments. The overall approach is consistent with the concept of Ambient Intelligence ${ }^{1,2}$. Potential applications of this architecture include: 
- Hospitals

- Airports / tube-train-bus stations

- Smart homes for elderly and disabled

- Roads and traffic related hazards

- Nuclear plants

- Stadium (e.g. for Olympic games 2010)

Examples of danger: terrorist threat to a public building (e.g. airport), health problem in a house or hospital.

Examples of resources: fire-brigade, police, doctors, ambulances, areas of restricted access, areas with specialized equipment, exits to buildings.

Examples of priorities: evacuate an area as soon as possible, do not move people and negotiate with criminals, approaching a house where there is a criminal without the criminal noticing the move, bringing ambulances through busy traffic.

Decision-making problem: how to react to unexpected/undesirable situations? How to make a decision when there are strongly conflicting aims (e.g. allowing the criminals to escape versus trying to capture them without harming the public).

We assume there is a main coordinating DM at the core of the DSS, which will have a "board of advisers" specializing on how to identify different dangers (e.g., fire, bomb threat, nuclear waste leakage) and proposing how to handle such dangers. The high-level view of the operational procedure is as follows:

1. situation detection

2. safety assessment (level of threat and objects involved)

3. define course of action (similar to a plan but may be at a more general level)

4. check with the DM and interact until there is consensus or there is no more need to look for a plan (maybe the threat disappears or all possible suggestions are bad).
5. If there is a plan agreed, give the steps to achieve the goal and monitor development. According to how things progress there may be a need to re-plan.

In this paper we focus on the first step of this process. We use a temporal logic language to represent causal relations of the world, which also assimilates dynamic and heterogeneous information. Decision-making support is performed by evaluating alternative explanations that can be used as predictions of potential future states of a world. A theory on preferences and associated algorithms are developed for arbitration of different explanations and of different advisers, which gives rise to the optimal decision for the situation.

Here we describe a theoretical framework to capture some key aspects of a decision support system. We focus in this paper on the logical core of it, which uses a simple representation of causal relationships (see Section 2) to build explanations for possible diagnosis of situations (see Section 3). These notions are illustrated by way of a scenario (Section 4) which is later used to explain how decision making can be supported by our system. Different preference criteria can be encoded to order explanations based on particular evidence. In the classical preference structures, the decision-maker is supposed to be able to totally compare the explanations. But certain situations, such as lack of information, uncertainty, ambiguity, and conflicting preferences, can lead to partial orders 3 between explanations. This problem is considered in Section 5 where we define a framework through which different preferences can be merged to make a preference criteria and select in between alternative explanations. This process is generalized in Section 6 for the case where a DM have to take decisions based on the advise of other subordinates.

\section{Causal Representation of the System}

An essential part of the decision making process deals with connecting current states of the system with potential scenarios for the DM to assess the situation and make appropriate judg- 
ments. We represent basic key features of the system and their inter-relation from a causal perspective.

States are partitioned into two classes: dependent $\left(\mathcal{S}_{D}\right)$ and independent $\left(\mathcal{S}_{I}\right) . \bar{S}$ is the opposite of $S$. An independent state does not depend causally on other states holding at the same time, whereas a dependent state can do so. An independent state can only be initiated by the occurrence of initiating or terminating events. A state $S$ will be co-independent if $\bar{S}$ is independent. Events will represent external influences to the system being modelled and will drive its internal change.

A specification of a system will be interpreted over a sequence of states, starting with the initial state $S_{0}: S_{0}, S_{1}, S_{2}, \ldots$ indexed by time. When the clock ticks at time $t$ the system leaves $S_{t}$ and the system is at state $S_{t+1}$. This new state is computed from $S_{t}$ by first applying any event $E_{i}$ such that $\operatorname{occurs}\left(E_{i}, t: t+1\right)$ (see Figure 2) and then applying the causal rules. An example of this will be given later when we introduce a practical scenario.

The following technical language, based on that of ${ }^{4}$, is used to represent our scenarios. We restrict ourselves to a propositional language where cause-effect relationships can be expressed directly in a simple way.

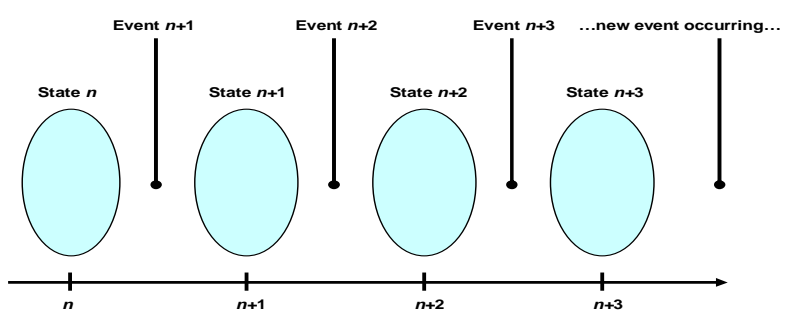

Fig. 2. Dynamic evolution of the system.

Definition 1 ( 4 page 3): There are two kinds of causal rule,

Same-time rules: $s_{1} \sqcap s_{2} \sqcap \cdots \sqcap s_{n} \rightsquigarrow s$

Next-time rules: $s_{1} \sqcap s_{2} \sqcap \cdots \sqcap s_{n} \rightsquigarrow \bigcirc s$ where each $S_{i}$ is an atomic state and $S \in \mathcal{S}_{D}$.

The intuitive interpretation is based on a set $\mathcal{S}$ of global states $S_{0}, S_{1}, S_{2}, \ldots$ and a time $t, t$ belong to the Natural numbers. By $S_{t}$ we mean the formulas which are true in the system at the state it is at time $t$, then $-s$ is the logical negation of a primitive state, $s_{1} \sqcap s_{2}$ is the conjunction of two primitive states. Same-time rules are such that if the antecedent is true at a time $t$ then the consequent is the effect of the causes given in the antecedent and that effect happens at the same time as the causes. In a Next-time rule the effect materializes at the next time $(t+1)$.

Same-time rules are required to be stratified. This is explained as follows ${ }^{4}$ :

1. A Stage 1 rule is a rule $S_{1} \sqcap S_{2} \sqcap \ldots \sqcap$ $S_{n} \rightsquigarrow S$, where $S_{1}, \ldots, S_{n}$ are all independent. In this case $S$ is said to be 1 dependent. (The independent states are called 0 -dependent.)

2. A Stage $k$ rule is a rule $S_{1} \sqcap S_{2} \sqcap \cdots \sqcap S_{n} \rightsquigarrow$ $S$, where each of $S_{1}, \ldots, S_{n}$ is at most $(k-1)$-dependent, and at least one of them is $(k-1)$-dependent. Then $S$ is said to be $k$-dependent. In this case we also say that $\bar{S}$ is co-k-dependent.

3. A set of same-time rules is stratified so long as for every rule in the set there is a number $k$ such that the rule is a Stage $k$ rule.

We provide an example of stratification at the end of Section 5.

Causal rules are applied to transition the system from state $S_{t}$ to the next state $S_{t+1}$ in order of $k$-dependency, i.e., first 1-dependent, then 2dependent and so on. See more details on this at ${ }^{4}$.

The language presented above can be extended by covering different alternatives (e.g., nonlinear, continuous time, delays, etc). We keep it simple enough to easily allow specification of dynamic systems and a tractable combination with other features of our system to be introduced later. 


\section{Alternative and Conflicting Explana- tions}

Decision-making is about evaluating alternatives, following a particular sequence of events the decision-maker is presented with a query and it is her/his task to assess the possible options to follow and make a decision. In this section we focus on the process of gathering the explanations available. At a later section we consider how to assess them.

Whenever a query about a particular state $S$ of the system is passed to the DM our system will assist the analysis by considering the causal structure leading to that particular state at a particular point in time. The assistance provided comes in the form of an explanation, i.e., it provides details on what events $e_{1}, e_{2}, \ldots$ are meaningful for that state to be reached and what causal laws $r_{1}, r_{2}, \ldots$ governing the system are being exercised when events in the system can cause a state $S$ to hold. We will call that a causal explanation. There may be more than one possible explanation for how the modeled system can reach $S$. These explanations can be consistent with each other in which case there is no conflict. But it may also be, due to the ambiguity or lack of information characteristic in real-time applications, that some of the explanations are contradictory or somehow antagonistic. Analyzing the quality of the explanations and why some of these are contradicting to each other is a difficult and time consuming task, to be avoided when a DM has to react to an imminent hazard. This part of our system is strongly related to previous developments in theory of temporal argumentation $(5,6,7,8)$.

We consider possible competing causal explanations $\left\langle c_{1}, s_{1}\right\rangle$ and $\left\langle c_{2}, s_{2}\right\rangle$ at time $t$, where $c_{1}$ is a causal structure (containing causal rules $r_{i}$ ) explaining why the system may reach state $s_{1}$ at time $t$ and $c_{2}$ an alternative causal structure (based on a set of causal rules $r_{j}$, disjoint with $r_{i}$ ) explaining why the system may reach state $s_{2}$ at time $t$. These causal explanations can be such that $s_{1}$ contradicts $s_{2}$ or contradicts the possibility that $c_{2}$ may exist. In this paper we focus on the way these possible causal explanations can be found and also on the potential scenarios that can cause two possible explanations to be mutually contradictory or undermining (similarly to rebuttal or undercutting arguments ${ }^{9}$ ).

\section{Airport Security}

Hazards can arise in many contexts and for many reasons. The following scenario describes situations involving hazards and possible explanations for them, each explanation suggesting different courses of action. Suppose an airport gathers information that a bomb may have been placed there. To react to this specific event and avoid potential disaster, the airport security system contacts national intelligence departments (NIDs) to collect the latest information on airport related terrorist threats. It also checks all surveillance information within the airport, such as video footage, and any reported suspected incidents. Lets assume no feedback has indicated attacks either being planned or underway. Facing with the bomb threat the airport authority has to make a hard decision on what to do next. While keeping the airport operating normally is critical, protecting human life from a bomb attack is certainly of paramount importance.

Lets assume the following simplified description of the decision making process. States are: bombAlert (a bomb alert has been issued), assesmRisk (assessment of real risk is needed), nidReq (NID is requested information on current threats), checkSRR (check current security related reports at the airport), nidRepOK (NID reports everything is normal), localInfoOK (security related information from the airport is normal), source1 (one source of information), source2 (another source of information), emergency (state of emergency is declared), suspectArrived (suspect is within the airport), passedC (suspect passed security checks), resultALowR (report indicates low risk), resultAHiMeR (report indicates medium or high risk).

Independent states are: bombAlert, resultALowR, resultAHiMeR, -resultALowR, and -resultAHiMeR. nidRepOK and localInfoOK are known to be true initially and all the rest false. For notation of states and events here we follow ${ }^{10}$ so $\operatorname{ingr}(S)$ denotes the event of 
and ingression to state $S$ occurring. The following events occur during the development of activities: occurs(ingr(bombAlert) at instant 1:2, occurs(ingr(source1) and occurs(ingr(-source2) at instant 3:4, occurs(ingr(suspectArrived) at instant 5:6, occurs(ingr(passedC) at instant 6:7, and finally occurs(ingr(resultALowR) and occurs(ingr(- resultAHiMeR) at instant 8:9. Lets assume we want to be able to investigate if an emergency should be declared, for example, at time 10 .

The following causal rules captures the basics of the scenario:

$$
\begin{aligned}
& \text { bombAlert } \rightsquigarrow \text { assesmRisk } \\
& \text { bombAlert } \rightsquigarrow \text { nidReq } \\
& \text { bombAlert } \rightsquigarrow \text { checkSRR } \\
& \text { suspectArrived } \rightsquigarrow \text {-localInfoOK } \\
& \text { suspectArrived } \sqcap \text { passedC } \rightsquigarrow \text { localInfoOK } \\
& \text { nidReq } \sqcap \text { resultALowR } \rightsquigarrow \text { nidRepOK } \\
& \text { nidReq } \sqcap \text { resultAHiMeR } \rightsquigarrow-\text { nidRepOK } \\
& \text {-nidRepOK } \rightsquigarrow \text { Oemergency } \\
& \text {-localInfoOK } \text { - Oemergency } \\
& \text { nidRepOK } \sqcap \text { localInfoOK } \sqcap \text { reliableSource } \rightsquigarrow
\end{aligned}
$$$$
\text { Oemergency }
$$$$
\text { nidRepOK } \sqcap \text { localInfoOK } \sqcap \text { unrelSource } \rightsquigarrow
$$$$
\bigcirc \text { - emergency }
$$

This KB is stratified as follows: the first five rules are at 0-level (only dependent on external events). The next two rules are at level 1 (dependent on the outcome of the previous level, 0$)$. The remaining rules are at level 2 (dependent on the outcome of the previous level, 1).

Based on the above rules, our Prolog-based prototype can extract the two possible competing explanations ( $A 1$ and $A 2)$ in relation to the declaration of emergencies:

$[\{$ nidRepOK $\sqcap$ localInfoOK $\sqcap$ reliableSource $\rightsquigarrow$ Oemergency\}, emergency]

$[\{$ nidRepOK $\sqcap$ localInfoOK $\sqcap$ unrelSource $\rightsquigarrow$ $\bigcirc$ - emergency\}, - emergency]

\section{Dynamic POSet Generation for Expla- nation Preference Handling}

Each time two or more explanations differ in the conclusion they have to be analyzed to decide which of them, if any, can be considered the most credible explanation(s). This section gives a more precise description on the process to compare and select competing explanations.

Typically the competing explanations can be arranged in a partial order and our approach will take that as the departing point aiming to generate a POSet out of the set of explanations being considered. In occasions it will be possible to order the options into a total order if there are a few options. Notice that we cannot ensure the POSet will be a lattice ${ }^{3}$ as we leave open the type of order relationships that can be used (provided the user gives an algorithm on how to use them) and therefore we cannot ensure the POSet will have a greatest lower bound or a lowest upper bound.

The process can be generically depicted as in Figure 3. The possible explanations are given to a specific module of the system which evaluates them based on domain specific and also general criteria to assess which is their relative strength.

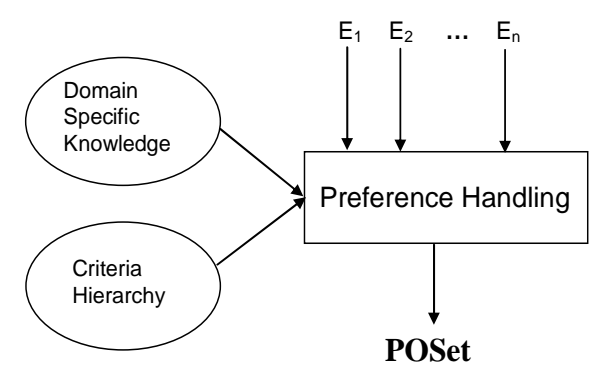

Fig. 3. Preference POSet generation.

A structure to evaluate the preference POSet of explanations can be syntactically constructed as follows.

Definition 2 a POSet evaluation structure is a 6-tuple $\langle A, B, C, D, Z, L\rangle$ where:

$A$ is a set of alternative explanations;

$B \in Z$ is a validity benchmark (what is the minimum value for an explanation to be valid/useful?); 


\section{$C$ a criteria to compare explanations and build a POSet. Here $C$ is triplet $\left\langle C_{1}, C_{2}, C_{3}\right\rangle$ where:}

$C_{1}$ is a set of criteria $\left\{C R_{1}, \ldots, C R_{m}\right\}$,

$C_{2}$ is a set of order meta-relationships defined over different criteria $\left\{M_{1}, M_{2}, \ldots, M_{n}\right\}$, and

$C_{3}$ is a meta-criterion defined over elements of $C_{1}$ using $C_{2}$. *

$D$ is the domain specific information;

$Z$ is a range of possible values used to rank the strength of the explanations; and

$L$ may be either a pair $(1,0)$ or $L=\emptyset$, depending on whether the POSet is a lattice or not. If the POSet is of type lattice then $L=(1,0)$ and symbol "1" represents the top element and " 0 " represents the bottom element, $\{$ "1", "0" $\} \subseteq Z$.

To illustrate the different elements of the structure defined above lets assume we have a structure $\langle A, B, C, D, Z,(1,0)\rangle$ defined over two possible explanations $a_{1}$ and $a_{2}$ : $\left\langle\left\{a_{1}, a_{2}\right\}\right.$, "acceptable", $\langle\{S, T, E P\},\{\triangleleft\},\{S \triangleleft$ $T \triangleleft E P\}\rangle,\{$ "excellent", "verygood", "good", "acceptable", "bad"\},("excellent","bad")). Lets assume $S$ stands for Specificity $\left({ }^{12},{ }^{8}\right), T$ for Trust and EP for Event Probability. " $\triangleleft$ " is the meta-relation 'precedence' which in the case of $S \triangleleft T \triangleleft E P$ indicates that our procedure will first apply $S$ and if that is not enough to decide then will apply $T$ and if that is not enough it will apply $E P$. This meta-relationship defines a total order of preference amongst the comparison criteria that can be applied to compare arguments. If after applying $S, T$, and $E P$ in the order established by $\triangleleft$ no explanation(s) is (are) established as the most preferred one(s) then all the explanations evaluated have equal strength. It may also be that one or both explanations $a_{i}$ and $a_{j}$ cannot be judged using any of these criteria so $a_{i}$ and $a_{j}$ will be said 'incomparable'. For the purpose of evaluating and comparing different explanations we will use a function $V\left(a_{i}, D\right)$ such that $V: A \times D \rightarrow Z$ which will measure the strength value of an explanation $a_{i} \in A$, possibly using information from the domain $D$, into a value of $Z$.

Airport scenario revisited: when a bomb alert is triggered several reassuring mechanisms are started as a consequence. Gathering information from different sources to confirm or discard the threat is essential. Information is gathered locally and also from specialized units (e.g., NID).

For the case of local information gathered we have two competing explanations: $\left\langle S_{1}\right.$, -localinfoOK $\rangle$ and $\left\langle S_{2}\right.$, localInfoOK $\rangle$ where:

$$
S_{1}=[\text { suspectArrived } \rightsquigarrow-\text { localInfoOK }]
$$

$S_{2}=[$ suspectArrived $\sqcap$ passed $C \rightsquigarrow \operatorname{localInfoOK}]$

Given they share the independent states which characterize these two possible developments they also share the list of interesting times to be investigated. As the set of causes related to explanation $S_{1}$ is entirely contained in $S_{2}$ and furthermore $S_{2}$ extends $S_{1}$ (is a "richer" explanation) then $S_{2}$ should be preferred to $S_{1}$. So the conclusion of the system will be that the alarm is off at 10 and the explanation is $S_{2}$. This conflict was decided purely on syntactical basis, i.e., the structure of the explanation allows us to take a decision based on the structure of the competing explanations. $S_{2}$ takes into account all that $S_{1}$ has to offer and also brings extra information on what happens after the presence of a suspect is detected. It states not only that the suspect has been detected but later on all the controls carried out over the individual has been negative in terms of identifying a cause of concern. Given that $S_{2}$ is preferable to $S_{1}$ by being more specific (i.e., according to criteria $S$ : $\left.S_{2}>_{S} S_{1}\right)$ the preference order is in this particular case a total order (left to right meaning going from up to down): $\left[V\left(S_{2}, D\right), V\left(S_{1}, D\right)\right]$ where $\mathrm{D}=$ "airport".

NID information is gathered as a risk estimation. For this particular problem it only matters if it is low enough for the threat to be considered.

${ }^{*}$ This is similar to Prakken's idea ${ }^{11}$ but use it to generate a POSet out of the preference criteria. 
If it is high or even medium then preventive measures have to be taken which is highlighted by the rules describing the scenario. We have two competing explanations: $\left\langle N_{1}\right.$, nidRepOK $\rangle$ and $\left\langle N_{2},-\right.$ nidRepOK $\rangle$ where:

$$
N_{1}=[\text { nidReq } \sqcap \text { resultALow } R \rightsquigarrow \operatorname{nidRepOK}]
$$

$N_{2}=[$ nidReq $\sqcap$ resultAHiMeR $\rightsquigarrow-n i d R e p O K]$

$N_{1}$ and $N_{2}$ are equally specific and the sources of evidence are equally strong (the same official organism) so according to the meta-criteria we consider next criteria $E P$ which (again this is domain dependent information) is based on the statistical information pointing out to the risk of attack being more likely the case $N_{2}$ by the criteria $E P$ and the resulting preference order is in this particular case a total order: $\left[V\left(N_{1}, D\right), V\left(N_{2}, D\right)\right]$.

Although confirmation from NIDs officials and 'in situ' evidence of danger suggest to ignore the call and even when the intuition of the DM tells everything is safe, information arrives that the source of the bomb alert can be trusted. If the DM is trying to collate all the supporting evidence from the system in terms of labelling a the situation as an emergency or not the system will consider the two possible contending explanations $A_{1}$ and $A_{2}$ for declaring an emergency or ignoring the threat. But at time 9 there is evidence for both, however the system will prefer explanations based on more reliable sources of information and hence $A_{1}$ will be preferred. Suppose in this context we have two competing explanations: $\left\langle A_{1}\right.$, emergency $\rangle$ and $\left\langle A_{2}\right.$, -emergency $\rangle$ where:

$A_{1}=[$ nidRepOK $\sqcap$ localInfoOK $\sqcap$ reliableSource

$\rightsquigarrow$ Oemergency]

$A_{2}=[$ nidRepOK $\sqcap$ localInfoOK $\sqcap$ unrelSource

$$
\rightsquigarrow \bigcirc \text {-emergency] }
$$

$A_{1}$ and $A_{2}$ are equally specific but the domain information in $D$ says the source of information informing about the possible threat is a reliable source of evidence. So according to the metacriteria we consider next criteria $T$ and $A_{1}$ is preferable to $A_{2}$ by the criteria $T$ and the resulting preference order is in this particular case a total order: $\left[V\left(A_{1}, D\right), V\left(A_{2}, D\right)\right]$.

Notice the examples above only considered two alternative explanations in each scenario but nothing forbids the existence of many possible explanations for both a thesis and its negation. The examples only involved total orders, see Figure 4, between arguments due to the short example used to illustrate the concepts as simply as possible:

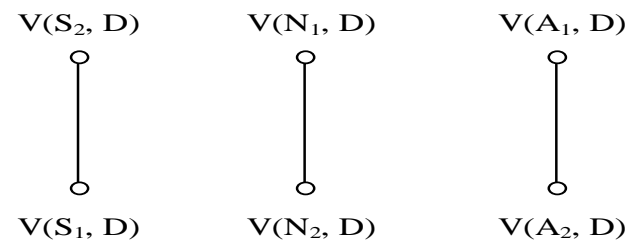

Fig. 4. Total order amongst arguments.

However any of those could have easily led to a non total order. Lets assume for the sake of illustration that another alternative to infer whether there is or not an emergency is that the Security Related Report is negative, meaning there is no suspicion that :

$$
\text { negative } S R R \rightsquigarrow \bigcirc-\text { emergency }
$$

then we have another contending explanation being activated and worth considering together with $A_{1}$ and $A_{2}$. Lets call it $A_{3}$, therefore: $\left[A_{3},-\right.$ emergency $]$. Lets also assume the conditions where the Security Related Report was conducted are not trustable (e.g., little time to gather serious feedback) and therefore it ranks low according to our comparison criteria. The final evaluation is $\left[V\left(A_{1}, D\right),\left\{V\left(A_{2}, D\right), V\left(A_{3}, D\right)\right\}\right]$ were $A_{2}$ and $A_{3}$ are equally ranked (both are considered unreliable inferences although for different reasons). Their POSets are shown in Figure 5.
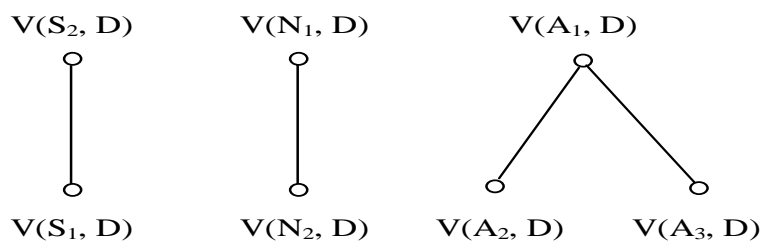

Fig. 5. Partial order amongst arguments. 
It should also be noticed that when we illustrated Definition 2 we considered a criterion to compare explanations based on a set of comparison criteria for arguments $C_{1}=\{S \triangleleft T \triangleleft E P\}$ which were ordered in a hierarchy of relevance $C_{2}=\{\triangleleft\}$. Then subsequent examples assumed the same elements $C_{1}$ and $C_{2}$. The metarelationship $\triangleleft$ is a total order but it does not have to be that way. We could have defined $C_{2}=\{\triangleleft\}$ and $C_{1}=\{S \triangleleft(T \backsim E P)\}$ meaning that $S$ has more relevance (has to be applied first) than both $T$ and EP. T and EP have equal relevance among themselves (any of them can be non-deterministically decided to be used first).

Figure 6 gives a basic description of the algorithm that is used to implement the dynamic POSet generation in the preference handling kernel of our system.

\section{Input: $\langle A, B, C, D, Z, L>$ and $V: A \times D \rightarrow Z$ as defined in Section 2}

Output: $S \subseteq A$ such that it ranks the highest according to $C$

1 repeat

$2 \quad$ Evaluate all elements of $A$ applying the first criteria as specified in $C_{3}$

$3 \quad$ Make $P$ the current POSet and $S$ the set of elements with highest $z \in Z$ associated such that for all $a \in S$, if $V(a)$ is its strength then $V(a)>B$

4 until ( $S$ is a singleton) or (all criteria on $C_{3}$ have been exhausted);

5 case of

$6 \quad S=\{\}$ : there is no acceptable

7 explanation

$8 \quad S$ is a singleton, $S=\{a\}$ : " $a$ " is the best explanation

$10 S=\left\{a_{1}, \ldots, a_{n}\right\}$ : elements of $S$ are

11 equally strong explanations

12 end case

Fig. 6. Weigthing explanations with a hybrid criteria.

The process above only considers what the outcome of only one DM. There can be occa- sions where more than one DM produces a decision and then another DM above them in the hierarchy will decide which advice is better. For example, DM1 takes: $\left\langle A, B, C^{1}, D, Z,(1,0)>\right.$ to produce a POSet $P_{1}$ and DM2 uses: $<A, B, C^{2}, D, Z,(1,0)>\left(\right.$ with $C^{1} \neq C^{2}$ ) producing a POSet $P_{2}$. One important problem to consider then is to sensibly merge $P_{1}$ and $P_{2}$ into a final $P$. This problem is addressed in the next section.

\section{Combination of Preferences}

The decision making process involving multiple decision makers (MDM) can be formulated as follows. We have a set of decision makers (voters) $\mathcal{V}=\left\{v_{1}, v_{2}, v_{3}, \cdots, v_{n}\right\}$, a set of alternative explanations $A=\left\{a_{1}, a_{2}, a_{3}, \cdots, a_{m}\right\}$, and a set $I$ of (individual) preferences where each $\alpha \in I$ is a partial order over a subset of $A$. For example, a preference may be $a_{2} a_{1} a_{3}, a_{4}$, or $a_{5} a_{4} a_{3} a_{2} a_{1}$. A preference may be obtained by a decision maker using a subset of criteria, or it may also be an overall decision by a separate decision maker.

The task of MDM is to find a total order that agrees maximally with all preferences in $I$. The preference relation considered here is qualitative, therefore we cannot use the standard Borda score based solution ${ }^{13,14}$. We present a probabilistic approach instead.

We treat a preference as a sequence, and consider the probability that one alternative is before another, based on individual preferences. We then apply the well known ORDERBYPREFERENCE algorithm ${ }^{15}$ which takes as input such probabilities and generate an approximately optimal total order (i.e., approximately maximally agrees with the preferences). Interested readers are invited to consult ${ }^{15}$ for more details on this algorithm. In the next section we focus on how to obtain such probabilities from given preferences.

\subsection{Contextual probability}

Let $\Omega$ be a set, $\mathcal{F}$ be a $\sigma$-field over $\Omega$. For example, $\mathcal{F}=2^{\Omega}$. The elements of $\mathcal{F}$ are called neighborhoods, and we can embed $\Omega$ in $\mathcal{F}$ so 
$\Omega \subseteq \mathcal{F}$. If there is a probability function $\mathcal{P}$ over $\mathcal{F}$ we have a probability space $\langle\Omega, \mathcal{F}, \mathcal{P}\rangle$. The contextual probability ${ }^{16}$ is $G: \mathcal{F} \rightarrow[0,1]$ such that, for $X \in \mathcal{F}$

$$
G(X)=\sum_{E \in \mathcal{F}} \mathcal{P}(E) f(X \cap E) / K
$$

where $K=\sum_{E \in \mathcal{F}} \mathcal{P}(E) f(E)$ is normalization factor and $f(X)$ is a non-negative measure of $X$ satisfying $f\left(X_{1} \cup X_{2}\right)=f\left(X_{1}\right)+f\left(X_{2}\right)$ if $X_{1} \cap X_{2}=\emptyset$, e.g., $f(X)=|X|$. For $t \in \Omega$ and $h \in \mathcal{F}$, if $t \in h$ we say $t$ is covered by $h$. Let $I$ be a finite sample of $\Omega$, where $N=|I|$. It has been shown that $G$ can be estimated based on $I$ as follows:

Lemma $1\left({ }^{16}\right)$ For $t \in \Omega$,

$$
\hat{G}(t)=\frac{1}{N \times K} \sum_{x \in I} \operatorname{cov}(t, x)
$$

where $\operatorname{cov}(t, x)=|\{E \in \mathcal{F}: t \in E, x \in E\}|$ is the number of all neighborhoods that cover both $t$ and $x$.

\subsection{Calculating cover for sequences}

Let $\mathcal{A}$ be a finite set of symbols (explanations). A sequence $\alpha$ is $\left\{s_{i}\right\}_{0}^{n}=\left\{s_{0}, s_{1}, \cdots, s_{n}\right\}$, or simply, $s_{0} s_{1} \cdots s_{n}$. We consider a data space, $\Omega$, of all sequences without repetition. Obviously $\Omega$ is finite.

Consider a sequence $\alpha$ and $a, b \in \alpha$. If $a$ comes before $b$ (from left to right) then $a \leq_{\alpha} b$. Consider two sequences $\alpha$ and $\beta$. If $\alpha$ can be obtained by removing some symbols from $\beta, \alpha$ is a subsequence of $\beta$, written by $\alpha \preceq \beta$. A neighborhood is a set of all sequences that share a single subsequence $\gamma$. We then say it is a neighborhood with respect to $\gamma$. Thus a neighborhood corresponds to a sequence. We write $\mathcal{F}$ for the set of all neighborhoods, or sequences. We can show that $\mathcal{F}$ is a $\sigma$-field. Incidentally the data space and the $\sigma$-field are the same.

A neighborhood of sequence $\alpha$ is a neighborhood with respect to a subsequence $\gamma$ of $\alpha$. Therefore the set of all neighborhoods of $\alpha$ is the set of all subsequences of $\alpha$, written by $N(\alpha)=\{\gamma: \gamma$ is a subsequence of $\alpha$. $\}$. Furthermore we write $N(\alpha, \beta)$ for the set of neighborhoods covering both $\alpha$ and $\beta$, which is in fact the set of all common subsequences of $\alpha$ and $\beta$. The algorithm in Figure 7 is designed to find all common subsequences.

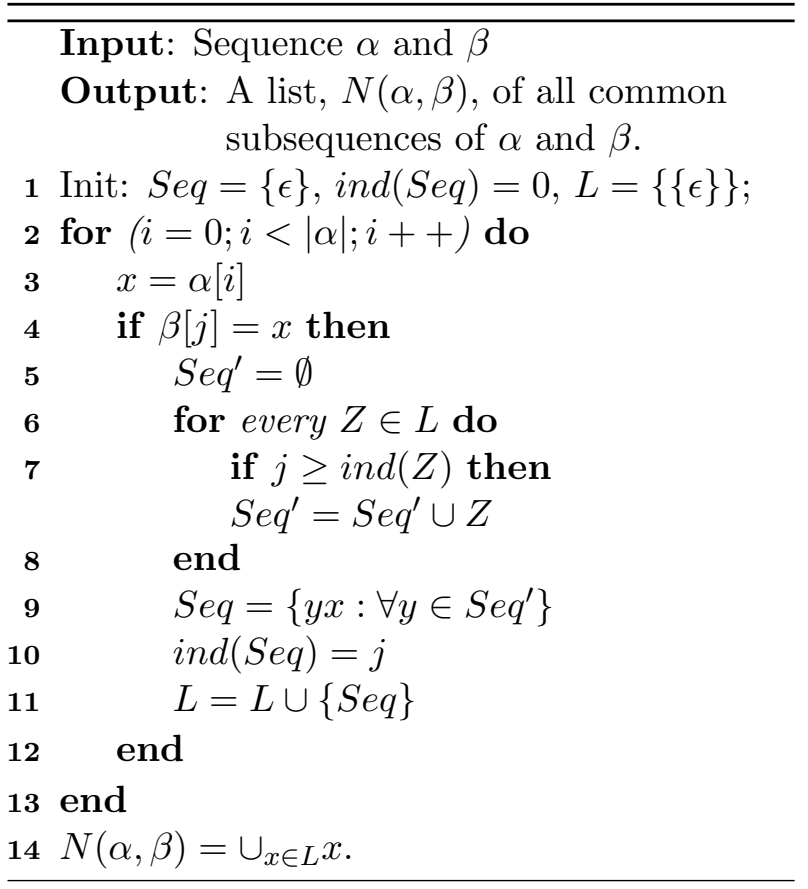

Fig. 7. Finding all common subsequences.

\subsection{The Probability that One Explanation is Before Another}

For any two explanations $a, b \in \mathcal{A}$ we consider a sequence $a b$. $a$ and $b$ can be regarded as singleton sequences.

According to Lemma 1 we have

$$
\hat{G}(a b)=\frac{1}{N K} \sum_{x \in I} \operatorname{cov}(a b, x)
$$

Recall that $K$ is a normalization factor independent of $a b, N$ is the number of sequences in $I$, and $\operatorname{cov}(a b, x)$ is the number of common subsequences of $a b$ and $x$. Similarly

$$
\hat{G}(b)=\frac{1}{N K} \sum_{x \in I} \operatorname{cov}(b, x)
$$

The probability that $a$ is before $b$ is then modeled by

$$
\hat{G}(a \mid b)=\frac{\hat{G}(a b)}{\hat{G}(b)}
$$




\subsection{An Example: Airport Scenario Revisited}

Suppose there are three independent advisers (ADVs): $a d v_{1}, a d v_{2}, a d v_{3}$, involved in the airport scenario to assist the decision maker and there are five alternative explanations $a, b, c, d, e$. The preferences by individual ADVs are $a d v_{1}$ : $a b c d e, a d v_{2}: a b, a c e d$, and $a d v_{3}: b c, a e d$. Assuming equal weighting of the ADVs we have a preference set $I=\{a b c d e, a b, a c e d, b c$, aed $\}$.

Consider a query sequence $q=a c$. To calculate $G(q)$ we need to calculate $\operatorname{cov}(q, x)$ for every $x \in I$, which is the number of all common subsequences of $q$ and $x$.

Consider $x=a b c d e$. The set of all subsequences of $x$ is $\{\emptyset, a, b, c, d, e, a b, a c, a d, a e, b c$, $b d, b e, c d, c e, d e, a b c, a b d, a b e, a c d$, ace, ade, bcd, bce, bde, cde, abcd, abce, abde, acde, bcde, abcde\}. The set of all subsequences of $a c$ is $\{\emptyset, a, c, a c\}$. Therefore $\operatorname{cov}(a c, a b c d e)=4$. Similarly we have $\operatorname{cov}(a c, a b)=2, \operatorname{cov}(a c, a c e d)=4$, $\operatorname{cov}(a c, b c)=2$, and $\operatorname{cov}(a c, a e d)=2$. Therefore $G(a c)=\frac{1}{N K}(4+2+4+2+2)=\frac{14}{N K}$.

We can similarly compute $G(a b), G(a d), G(a e)$, $G(b c), G(b d), G(b e), G(c d), G(c e)$, and $G(d e)$. Feeding this $G$ values to the OrderbyPreferENCE algorithm will produce a total order over the alternative explanations, which is guaranteed to be approximately optimal. This total order can be used to make a consensuated decision.

\section{Related Work}

Some related systems like ${ }^{8}$ and ${ }^{7}$ focused already on temporal argumentation systems but both are based on one DM and one fixed preference criteria. The former uses specificity whilst the second only counts the number of arguments in favour and opposed to a claim. We believe different contexts and different DMs will impose different criteria and therefore for a system to be of practical use that component of the system has to allow more flexibility.

Research and endeavor towards disaster management have been undertaken and exercised in various initiatives under different banners. For example, the United Nation started the International Decade for Natural Disaster
Reduction (IDNDR) programme in late 1980s and the follow-up International Strategy for Disaster Reduction (ISDR) programme in late 1990s ${ }^{17}$. The application of information technologies to enhance disaster management ${ }^{18}$ has been a constant centre of research, which explores the critical and evolving role of IT and its infrastructure, in particular, the communication and information sharing. Recently using agent technologies for disaster management has got momentum with a number of agent-based disaster management simulation systems being developed ${ }^{19},{ }^{20}$. These systems provide disaster management experience for DMs through training and experiments; and technically they concentrate on the co-ordination of agents.

Instead we focus here on real-time support for DMs. Our framework assist DMs to evaluate situations and make decisions as a threat is unfolding. The system can be used to detect particular events and warn on possible threats as well as to predict a possible outcome and give an explanation associated with the conclusion.

The system can work in two modalities, by channeling all knowledge and outcome to one Decision Maker(s) which will make a final decision or by combining several partial decisions from teams of decision makers. The advice to the Decision Maker(s) is based on decision criteria which can be adapted to different domains.

\section{Conclusions}

We present a framework that combines important features to the decision-making process. The use of causality reasoning based on the temporal evolution of a scenario provides a natural way to chain meaningful events and possible states of the system. Allowing the specification of possibly conflicting situations also brings a very typical element in these domains as there are usually different ways to analyze a problem, different possibly competing strategies to follow and it is often the case that information originating in different sources can be inconsistent. A decision procedure to decide on those conflicting situations has been explained which serves as a decision making framework suitable to one $\mathrm{DM}$ or to a hierarchy of DMs. 


\section{References}

1. J. C. Augusto. Ambient intelligence: The confluence of pervasive computing and artificial intelligence. In Alfons Schuster, editor, Intelligent Computing Everywhere, pages 213-234. Springer Verlag, 2007.

2. J. C. Augusto, J. Liu, and L. Chen. Using ambient intelligence for disaster management. In Springer, editor, Proceedings of 10th International Conference of KES2006 (Knowledge-Based Intelligent Information and Engineering Systems), Part II, volume 4252 of Lecture Notes in Computer Science, pages 171-178, Bournemouth, UK, October 2006.

3. James L. Hein. Discrete Structures, Logic, and Computability. Jones and Bartlett, 2002.

4. A. Galton and J. C. Augusto. Two approaches to event definition. In A. Hameurlain, R. Cicchetti, and R. Traunmüller, editors, Proceedings of DEXA 2002, pages 547-556. LNCS Vol. 2453 Springer Verlag, 2002.

5. G. Ferguson and J. Allen. Trips: An integrated intelligent problem-solving assistant. In Proceedings of 15th National Conference on AI, pages 567573, 1998.

6. Carlos Chesñevar, Ana Maguitman, and Ronald Loui. Logical models of argument. ACM Computing Surveys, 32(4):337-383, 2000.

7. John Fox and Subrata Das. Safe and Sound, Artificial Intelligence in Hazardous Applications. AAAI Press/MIT Press, 2000.

8. J. C. Augusto and Guillermo R. Simari. Temporal defeasible reasoning. Knowledge and Information Systems, 3(3):287-318, 2001.

9. J.L. Pollock. Cognitive Carpentry. A Blueprint for How to Build a Person. MIT Press, 1995.

10. A. Galton. Eventualities. In M. Fisher, D. Gabbay, and L. Vila, editors, Handbook of Temporal
Reasoning in Artificial Intelligence, pages 25-58. Elsevier, 2005.

11. H. Prakken. Logical Tools for Modelling Legal Argument. Kluwer Academic Publishers, Dordretch, 1997.

12. F. Stolzenburg, A. Javier García, C. Chesñevar, and G. Simari. Computing generalized specificity. Journal of Applied Non-Classical Logics, 13(1):87-113, 2003.

13. Kenneth J. Arrow. Social Choice and Individual Values. John Wiley and Sons, New York, 1963.

14. T.N. Tideman. Independence of clones as a criterion for voting rules. Social Choice and Welfare, 4:185-206, 1987.

15. W. W. Cohen, R. E. Schapire, and Y. Singer. Learning to order things. Journal of Artificial Intelligence Research, 10:243-270, 1999.

16. H. Wang and W. Dubitzky. A flexible and robust similarity measure based on contextual probability. In Proceedings of IJCAI'05, pages 27-32, 2005.

17. ISDR. International Strategy for Disaster Reduction (ISDR), $2006 . \quad$ URL: www.fire.uni-freiburg.de/programmes/ un/idndr/idndr.html.

18. S. Chien, B. Cichy, A. Davies, D. Tran, G. Rabideau, R. Castano, R. Sherwood, D. Mandl, S. Frye, S. Shulman, J. Jones, and S. Grosvenor. An autonomous earth-observing sensorweb. IEEE Intelligent Systems, 20(3):16-24, 2006.

19. N. Schurr, J. Marecki, P. Scerri, J. P. Lewis, , and M. Tambe. The defacto system: Training tool for incident commanders, volume Proceedings of IAAI'05. AAAI Press, 2005.

20. J. Buford, G. Jakobson, L. Lewis, N. Parameswaran, and P. Ray. D-AESOP: a situation aware BDI agent system for disaster situation, volume Proceedings of the First International Workshop on Agent Technology for Disaster Management. Japan, 2006. 